\title{
Erratum
}

\section{Crocetin protects against cardiac hypertrophy by blocking MEK-ERK1/2 signalling pathway}

\author{
Jun Cai ${ }^{a, b}$, Fang-Fang $\mathbf{Y i}^{\text {b }}$, Zhou-Yan Bian ${ }^{\text {c, d }}$, Di-Fei Shen ${ }^{c, d}$, Long Yang ${ }^{b}$, \\ Ling Yan ${ }^{c, d}$, Qi-Zhu Tang ${ }^{\text {c, d }}$, Xin-Chun Yang ${ }^{\text {b }}$, Hongliang Li ${ }^{\text {c, d }}$ \\ ${ }^{a}$ Cardiovascular Research Center, Massachusetts General Hospital, and Harvard Medical School, Boston, MA, USA \\ ${ }^{b}$ Department of Cardiology, Beijing Chaoyang Hospital, Affiliate of Capital Medical University, Beijing, China \\ ${ }^{c}$ Department of Cardiology, Renmin Hospital of Wuhan University, Wuhan, China \\ ${ }^{d}$ Cardiovascular Research Institute of Wuhan University, Wuhan, China
}

There is a correction concerning the correspondence details of an article published in a previous issue of the Journal of Cellular and Molecular Medicine [1]. The correspondence $\left({ }^{*}\right)$ symbol was attributed to both X-C Yang and H Li's names in the authors list. However, corresponding details were only presented for X-C Chang. H Li's corresponding details are as follows:

Hongliang Li, M.D., Ph.D.,

Cardiovascular Research Institute of Wuhan University,

JieFang Road 238, Wuhan 430060, China.

Tel./Fax: (86)-27-88083385

E-mail: hongliang.li@ymail.com

We apologise for this error.

[1] Cai J, Yi F-F, Bian Z-Y, et al. Crocetin proects against cardiac hypertrophy by blocking MEK-ERK1/2 signalling pathway. J Cell Mol Med. 2009; 13: $909-25$. 